\title{
Spontaneous and Forced Exercise Promotes Cognitive Function and Expression of GAP-43 and NT-3 in the Hippocampus of Aged Rats
}

\author{
Song-Hee Cheon, PT, PhD ${ }^{1)}$, Hyun-Mo Koo, PT, PhD²) \\ 1) Department of Physical Therapy, Youngsan University \\ 2) Department of Physical Therapy, Kyungsung University: 309 Suyeong-ro, Daeyeon-dong, Nam-gu, \\ Busan, Republic of Korea. TEL: +82 51-663-4872, FAX: +82 51-624-1394, E-mail: hmkoo@ks.ac.kr
}

\begin{abstract}
Purpose] The aim of this study was to investigate differences in cognitive function and expression of GAP-43 and NT-3 on the aged rat hippocampus in spontaneous (wheel running) and forced (treadmill) exercise groups. [Subjects and Methods] 45 male Sprague-Dawley rats (48-week-old aged rats) were randomly divided into 3 groups: namely, the controls $(n=15 ; \mathrm{CON})$, voluntary exercise group $(\mathrm{n}=15 ; \mathrm{VE})$, and forced exercise group $(\mathrm{n}=15 ; \mathrm{FE})$. Each exercise was applied for five days per week for 8 weeks, and the object recognition and object location tests were conducted in both the control and exercise groups after 8 weeks. In addition, Western blotting and immunohistochemistry were used to evaluate GAP-43 and NT-3 expression. [Results] There was significant improvement in the spontaneous exercise group and more improvement in the forced exercise group in the object location and object recognition tests for evaluation of cognitive function. Moreover, these results were similar to the results in GAP-43 and NT-3 immunohistochemistry in the hippocampus. [Conclusion] The present study suggests that forced exercise is a useful strategy for preventing the loss of cognitive function in the elderly. Key words: Exercise, GAP-43, NT-3
\end{abstract}

(This article was submitted Sep. 14, 2012, and was accepted Nov. 7, 2012)

\section{INTRODUCTION}

Aging can induce improper functioning of cognitive mechanisms by altering the function of the hippocampus, which is known to play a pivotal role in learning and memory ${ }^{1)}$. The hippocampus performs crucial functions in the performance of spatial tasks, and the activation of hippocampal cells is critical with regard to spatial representation. Additionally, the hippocampus, and in particular the neurotrophic factor in the dentate gyrus, is important in regard to the maintenance and improvement of learning and memory functions; reduced stimulation of the neurotrophic factor has been shown in previous studies to lead to a decline in nerve cell formation ${ }^{2)}$.

Neurotrophins (NTs) play an important role in the development and maintenance of the peripheral and central nervous system. The NTs can induce a variety of biological influences as a result of combination with specific tyrosine kinase receptors (trks) with high affinity ${ }^{3}$. As NT-3, in particular, among the NTs combines with trkC, due to the substantial amount of trkC distributed throughout the hippocampus, the expression level of NT-3 tends to be fairly high ${ }^{4}$. Growth-associated protein-43 (GAP-43) is an intracellular protein than contributes to the formation and reorganization of the synapses by inducing axonal development during the embryonic developmental period ${ }^{5)}$. This protein is also involved in axonal regeneration and sprouting at adulthood, and when the axon grows actively, it is expressed abundantly in the growth cone, sprouting axons in the central nervous system of adults, and presynaptic nerve terminals ${ }^{6}$.

The fact that exercise improves overall physical health as well as cognitive function has been demonstrated in several previous studies. Hillman et al. ${ }^{7}$ reported previously that aerobic exercises improve learning ability and form new nervous system structures by increasing the secretion of neurological substances associated with synaptic plasticity, and Trejo et al. ${ }^{8)}$ previously noted that increases in cell proliferation and survival in the hippocampal dentate gyrus are the most consistently observed results of sports training, such as running.

In an animal model, spontaneous exercises using a running wheel and forced exercise using a treadmill have been studied via a variety of methods ${ }^{9)}$. Wheel running is generally regarded as a type of voluntary exercise in animal models, and it does not activate systemic stress. However, there has been some controversy regarding the effects of both types of exercises on the nervous system. Albeck et al. ${ }^{10)}$ previously reported improvements in cognitive function by using treadmill exercises, a type of forced exercise, in aging rats; additionally, Kim et al. ${ }^{11)}$ noted previously that treadmill exercise can be very useful in preventing impaired memory functions in aging rats. However, it should be noted that O'Callaghan et al. ${ }^{12)}$ determined that even though forced exercise can result in better exercise performance, because of increased stress, the results of voluntary exercises can be decreased. Additionally, Yuede et al. ${ }^{13)}$ reported that 
Table 1. Result of the object location test and the object recognition test in each group

\begin{tabular}{lccc}
\hline \multirow{2}{*}{ Group } & \multicolumn{3}{c}{ Exprolation Time (\%) } \\
\cline { 2 - 4 } & CON & VE & FE \\
\hline Object location test & $60.32 \pm 2.25 \%$ & $68.86 \pm 2.47 \%^{*}$ & $75.35 \pm 1.83 \%^{*}$,\# \\
Object recognition test & $58.65 \pm 2.53 \%$ & $69.38 \pm 2.16 \%^{*}$ & $72.45 \pm 2.01 \%^{*}$ \\
\hline
\end{tabular}

Entries are exploration ratio $\pm \mathrm{SE}$ ([time with new object/time with old+time with new object] $\times 100$ ) of 15 subjects per group in the object location test. Data were analyzed via one-way ANOVA. Object location test. Significant differences were determined to exist by ANOVA $(\mathrm{F}=11.697, \mathrm{p}<0.05) .{ }^{*} \mathrm{p}<0.05$ vs. control. $\# \mathrm{p}<0.05$ vs. VE. Object recognition test. ANOVA $(\mathrm{F}=10.398, \mathrm{p}<0.05)$ showed significant differences. ${ }^{*} \mathrm{p}<0.05$ vs. control.

as a result of using voluntary exercises and forced exercises in mice suffering from senile diseases such as Alzheimer's disease, voluntary exercises were found to impair memory and allow the disease to progress.

Therefore, the principal objective of this study was to assess the effects of exercise types on the nervous system through the patterns of GAP-43 and NT-3 expression in the hippocampus, which plays a pivotal role in spatial memory and learning, after determining the effects of forced and voluntary exercises on short-term memory and spatial memory in aging rats.

\section{SUBJECTS AND METHODS}

Forty-five male Sprague-Dawley rats (48-week-old aged rats) weighing between $250 \mathrm{~g}$ and $300 \mathrm{~g}$ were used and maintained with a 12-hour on/12-hour off light/dark cycle with ad libitum access to food and water. All the experiments were conducted in accordance with the protocols established by the Daegu University Animal Experiment Committee based on the NIH Guidelines for the Care and Use of Laboratory Animals (NIH publication, 1996). The animals were randomly divided into 3 groups: namely, the controls $(\mathrm{n}=15 ; \mathrm{CON})$, voluntary exercise group $(n=15 ; V E)$, and forced exercise group $(n=15$; FE). A 12-hour darkness period in a breeding field with a running wheel apparatus with a diameter of $32 \mathrm{~cm}$ and a width $10 \mathrm{~cm}$ was used to test the VE group. By modifying the methods of Jenson et al. ${ }^{14)}$, the rats of the FE group were forced to run on the motor-driven treadmill. The treadmill was used at a speed of $16 \mathrm{~m} / \mathrm{min}$ during the dark period, and the total exercise time was set each day based on according to the average of the total distance the VE group moved in one day in the dark period. Each exercise was applied for five days per week for 8 weeks, and object recognition and object location tests were conducted in both the control and exercise groups after 8 weeks.

The animals were sacrificed via anesthesia with a mixture of $2 \mathrm{~mL} / \mathrm{kg} 50 \%$ Zoletil and 50\% Xylazine hydrochloride. The brains were removed for immunohistochemistry and Western blot analysis.

The results were expressed as means \pm standard error (SE). All experiments were analyzed via analysis of variance, and some experiments were analyzed via comparisons of the treatment mean with the controls via the Bonferroni-Dunn test. Differences were regarded as statistically significant at a $\mathrm{p}$ of $<0.05$.

\section{RESULTS}

The results of the object location test for the comparison group, VE group, and $\mathrm{FE}$ group were $60.32 \pm 2.25 \%$, $68.86 \pm 2.47 \%$ and $75.35 \pm 1.83 \%$, respectively. One-way analysis of variance was used to verify significance, and the significant probability indicated a significant difference of 0.000 . The results of the post-analysis showed significant differences between both the VE and FE groups evidenced relative to the comparison group, and significant differences were also found between the VE and FE groups (Table 1).

The results of the object recognition test for the comparison group, VE group, and $\mathrm{FE}$ group were 58.65 $\pm 2.53 \%$, $69.38 \pm 2.16 \%$, and $72.45 \pm 2.01 \%$, respectively. One-way analysis of variance was used to verify significance, and the significant probability indicated a significant difference of 0.000 . The results of the post-analysis showed significant differences between both the VE and FE groups evidenced relative to the comparison group, however, even though a difference is shown in the figure, no significant difference was noted between the VE and FE groups (Table 1).

The GAP-43 expression pattern in the hippocampus was observed after extracting the brains of aging rats that had been subjected to experimental measurements of GAP-43 expression patterns. As a result, GAP-43 expression was shown to be increased in both the VE and FE groups relative to the comparison group, but statistical significance was verified only in the FE group, and a significant difference was noted between the VE and FE groups (Table 2) (Fig. 1).

The NT-3 expression pattern in the hippocampus was assessed after extracting the brains of aging rats that had been subjected to a complete experimental measurement of the NT-3 expression pattern. The results showed that NT-3 expression was increased in both the VE and FE group by more than in the comparison group, but only the FE group showed a significant difference from the comparison group (Table 2) (Fig. 1).

\section{DISCUSSION}

Structural and physiological damage of the hippocampus, which processes memory, can occur as a result of aging, and are known particularly to lead to a reduction in spatial memory. Memory functions can be improved by exercise.

The principal objective of this study was to evaluate the effects of voluntary exercise and forced exercise on the 
CON

A

GAP-43

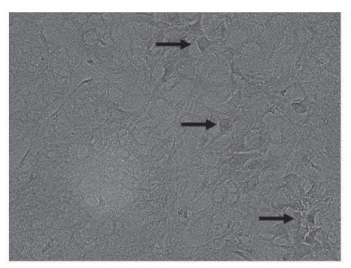

B

NT-3

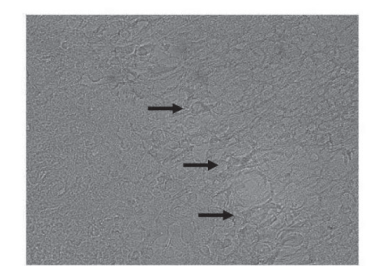

VE
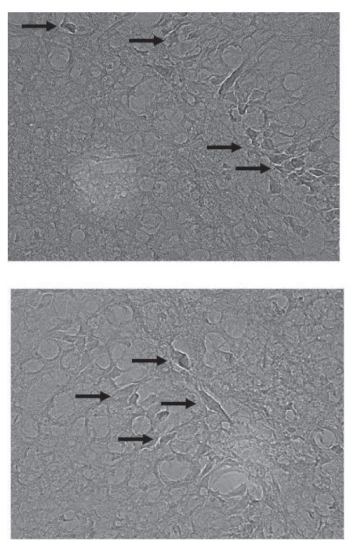

FE
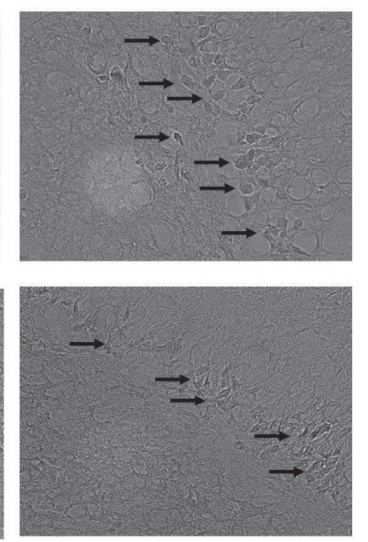

Fig. 1. Immunomicrographs $(\times 100)$ in the hippocampus. The expression of GAP-43 and NT-3 was detected via immunohistochemistry with anti-GAP-43 and anti-NT-3 antibodies, respectively, as described in the Subjects and Methods section. A. GAP-43; B. NT-3.

Table 2. GAP-43 and NT-3 expression in the hippocampus

\begin{tabular}{lccc}
\hline \multirow{2}{*}{ Group } & \multicolumn{3}{c}{ Relative optical density (percentage of control) } \\
\cline { 2 - 4 } & CON & VE & FE \\
\hline GAP-43 & $100 \pm 4.27$ & $111.56 \pm 4.28$ & $128.52 \pm 2.20^{* \#}$ \\
NT-3 & $100 \pm 2.82$ & $114.96 \pm 2.55$ & $125.54 \pm 3.30^{*}$ \\
\hline
\end{tabular}

The amounts of GAP-43 and NT-3 were detected via Western blotting with anti-GAP-43 and anti-NT-3, respectively, as described in the Materials and Methods section. Each example shown is representative of three experiments. The values represent the means \pm SE of three independent experiments conducted in triplicate dishes. ${ }^{*} \mathrm{p}<0.05$ vs. control. $\# \mathrm{p}<0.05$ vs. VE.

deterioration of hippocampal functions due to aging. The object location and recognition tests were used to compare memory ability, which is the main function of the hippocampus, after 8 weeks. Our results show that memory was significantly improved in both the VE group and FE group relative to the control group. However, the FE group showed a greater increase than was observed in the VE group, and a statistically significant difference was noted in the object location test between the VE group and FE group.

Spatial memory is the ability to cognitively construct the image of a location in space and to effectively explore that environment; thus, the object location test is very relevant to spatial working memory ${ }^{15)}$. The clear distinction observed between the VE and FE groups in the object location test as compared with the object recognition test can be interpreted as indicating that forced exercise has a more positive influence on the hippocampus, which plays an important role in spatial memory.

Burns et al. ${ }^{16}$ previously reported that the level of exercise is related to hippocampal volume in Alzheimer's disease patients and that forced exercise has the benefit of reducing hippocampal atrophy to some degree. In addition, Hayes et al. ${ }^{17)}$ reported that forced exercises combined with stress reduce the traumatic brain injury region by more than voluntary exercises and that the effects of stress as a single factor are inadequate in terms of nervous system recovery.

Many previous studies have reported that exercise potentiates neuronal formation, long-term potentiation, and synaptic plasticity ${ }^{18}$. Exercises that potentiate cognitive function produce an increase in hippocampal neuron formation. In the present study, GAP-43 and NT-3 were used to determine the effects of voluntary and forced exercise on hippocampal structure. The results showed that expression of GAP-43 and NT-3 increased by more in the FE group compared with the VE group and in the VE group than in the control group. However, statistically significant differences were found between the FE group and the control group, and significant differences between the VE group and FE group were identified only in GAP-43 expression.

GAP-43 is a phosphate that depends on the neurotrophin found in the axonal ending and neuron growth cones and is related to increased neurotransmitter release during long-term potentiation and GAP-43 phosphorylation ${ }^{19)}$. Therefore, the significant increase in the FE group is considered to indicate that forced exercise stimulates hippocampal neuronal plasticity more than voluntary exercise, which corresponds to the results of the object location test, a hippocampus-dependent task.

Even though NT-3 potentiates action potential-dependent excitatory synaptic activity (sEPSCs) and increases GAP-43 expression, NT-3 expression involves a very complicated process, which changes according to its subordinate activities $^{20)}$. At this point, the significant increase in the FE group has similarities to the expression pattern of GAP-43. This indicates that forced exercises function as afferent stimuli. However, Dishman et al. ${ }^{21)}$ emphasized that more information will be necessary to gain sufficient insight into the neurophysiological effects of exercise, including the effects of exercises in relation to various diseases. Although the results of this study demonstrated that forced exercises increase hippocampal function more than voluntary exercises, the study was limited by the relatively short 
exercise time imposed herein. However, the results of this study showed that forced exercise may constitute a more useful strategy than voluntary exercise in preventing agingrelated cognitive function losses.

\section{ACKNOWLEDGEMENT}

This research was supported by a Kyungsung University Research Grant in 2011.

\section{REFERENCES}

1) Mattson MP, Magnus T: Ageing and neuronal vulnerability. Nat Rev Neurosci, 2006, 7: 278-294. [Medline] [CrossRef]

2) van Praag $\mathrm{H}$, Shubert $\mathrm{T}$, Zhao $\mathrm{C}$ et al: Exercise enhances learning and hippocampal neurogenesis in aged mice. J Neurosci, 2005, 25:8680-8685. [Medline] [CrossRef]

3) Bothwell M: Functional interactions of neurotrophins and neurotrophin receptors. Annu Rev Neurosci, 1995, 18: 223-253. [Medline] [CrossRef]

4) Drake CT, Milner TA, Patterson SL: Ultrastructural localization of fulllength trkB immunoreactivity in rat hippocampus suggests multiple roles in modulating activity-dependent synaptic plasticity. J Neurosci, 1999, 19 8009-8026. [Medline]

5) Hrdina P, Faludi G, Li Q, et al: Growth-associated protein (GAP-43), its mRNA, and protein kinase $\mathrm{C}$ ( $\mathrm{PKC})$ isoenzymes in brain regions of depressed suicides. Mol Psychiatry, 1998, 3: 411-418. [Medline] [CrossRef]

6) Benowitz LI, Routtenberg A: GAP-43: an intrinsic determinant of neuronal development and plasticity. Trends Neurosci, 1997, 20: 84-91. [Medline] [CrossRef]

7) Hillman $\mathrm{CH}$, Erickson $\mathrm{KI}$, Kramer AF: Be smart, exercise your heart: exercise effects on brain and cognition. Nat Rev Neurosci, 2008, 9: 58-65. [Medline] [CrossRef]

8) Trejo JL, Carro E, Torres-Aleman I: Circulating insulin-like growth factor I mediates exercise-induced increases in the number of new neurons in the adult hippocampus. J Neurosci, 2001, 21: 1628-1634. [Medline]

9) Ang ET, Gomez-Pinilla F: Potential therapeutic effects of exercise to the brain. Curr Med Chem, 2007, 14: 2564-2571. [Medline] [CrossRef]
10) Albeck DS, Sano K, Prewitt GE: Mild forced treadmill exercise enhances spatial learning in the aged rat. Behav Brain Res, 2006, 168: 345-348. [Medline] [CrossRef]

11) Kim SE, Ko IG, Kim BK, et al.: Treadmill exercise prevents aging-induced failure of memory through an increase in neurogenesis and suppression of apoptosis in rat hippocampus. Exp Gerontol, 2010, 45: 357-365. [Medline] [CrossRef]

12) O'Callaghan RM, Ohle R, Kelly AM: The effects of forced exercise on hippocampal plasticity in the rat: A comparison of LTP, spatial- and non-spatial learning. Behav Brain Res, 2007, 176: 362-366. [Medline] [CrossRef]

13) Yuede CM, Zimmerman SD, Dong H et al: Effects of voluntary and forced exercise on plaque deposition, hippocampal volume, and behavior in the Tg2576 mouse model of Alzheimer's disease. Neurobiol Dis, 2009, 35: 426-432. [Medline] [CrossRef]

14) Jeneson JA, de Snoo MW, Verlinden NA, et al.: Treadmill but not wheel running improves fatigue resistance of isolated extensor digitorum longus muscle in mice. Acta Physiol (Oxf), 2007, 190:151-161. [Medline] [CrossRef]

15) Ennaceur A, Neave N, Aggleton JP: Spontaneous object recognition and object location memory in rats: the effects of lesions in the cingulate cortices, the medial prefrontal cortex, the cingulum bundle and the fornix. Exp Brain Res, 1997, 113: 509-519. [Medline] [CrossRef]

16) Burns JM, Cronk BB, Anderson HS, et al.: Cardiorespiratory fitness and brain atrophy in early Alzheimer disease. Neurology, 2008, 71: 210-216. [Medline] [CrossRef]

17) Hayes K, Sprague S, Guo M, et al.: Forced, not voluntary, exercise effectively induces neuroprotection in stroke. Acta Neuropathol, 2008, 115 289-296. [Medline] [CrossRef]

18) Olson AK, Eadie BD, Ernst C, et al: Environmental enrichment and voluntary exercise massively increase neurogenesis in the adult hippocampus via dissociable pathways. Hippocampus, 2006, 16: 250-260. [Medline] [CrossRef]

19) Tejero-Díez P, Rodriguez-Sanchez P, Martin-Cofreces NB, et al.: bFGF stimulates GAP-43 phosphorylation at ser41 and modifies its intracellular localization in cultured hippocampal neurons. Mol Cell Neurosci, 2000, 16: 766-780. [Medline] [CrossRef]

20) Gómez-Pinilla F, Ying $Z$, Roy RR, et al.: Afferent input modulates neurotrophins and synaptic plasticity in the spinal cord. J Neurophysiol, 2004, 92: 3423-3432. [Medline] [CrossRef]

21) Dishman RK, Berthoud HR, Booth FW, et al.: Neurobiology of exercise. Obesity (Silver Spring), 2006, 14: 345-356. [Medline] [CrossRef] 